\section{Radiographic Evidence of Hip Joint Recovery in Patients with Ankylosing Spondylitis after Treatment with Anti-tumor Necrosis Factor Agents: A Case Series}

\section{To the Editor:}

Ankylosing spondylitis (AS) is a chronic inflammatory disease of the axial skeleton and large peripheral joints. The efficacy of tumor necrosis factor (TNF) antagonists in the treatment of AS has been documented in several metaanalyses of randomized trials, in which significant improvements in disease activity were observed ${ }^{1}$. However, there is no evidence that anti-TNF- $\alpha$ agents prevent radiographic progression in patients with AS. In rheumatoid arthritis (RA), anti-TNF- $\alpha$ agents can prevent bone erosion and radiographic progression by suppressing osteoclast activity ${ }^{2,3}$. Unlike new bone formation in the axial spine, synovial inflammation within the hip joint induces bone erosion and subsequent joint destruction ${ }^{4,5}$ in AS. Additionally, the histological appearance of the synovial membrane of the hip joint in patients with AS is similar to that observed in $\mathrm{RA}^{6}$. These results suggest that both osteoproliferative and osteodestructive changes may occur in parallel at different sites in AS. In the present case series, we reviewed 6 patients with AS whose hip joint space increased after anti-TNF- $\alpha$ treatment.

The 6 patients had been treated with TNF inhibitors because of high disease activity despite treatment with nonsteroidal antiinflammatory drugs or disease-modifying antirheumatic drugs. The expert musculoskeletal radiologist and 1 experienced rheumatologist confirmed hip joint space changes by 2 different methods using anteroposterior radiographs of the pelvis before and after anti-TNF- $\alpha$ treatment. The Bath Ankylosing Spondylitis Radiology Index (BASRI) hip score ${ }^{7}$ and the hip joint space width (JSW) estimation method were used ${ }^{8}$. The second method measures the JSW as the average distance between the acetabulum and femoral head at 3 sites. Statistical analysis was performed using SPSS (version 17 for Windows). Intraobserver and interobserver agreement was estimated by calculating the intraclass correlation coefficient (ICC). The values for JSW were high (intraobserver ICC $=0.98,95 \%$ CI 0.94-0.99; interobserver ICC $=0.93,95 \%$ CI $0.28-0.99)$. The Wilcoxon signed-rank test was used to compare the mean differences in the JSW because of the small number of cases.

The BASRI score improved from 3 points to 2 points in the 6 patients after anti-TNF- $\alpha$ therapy. Figure 1 shows the hip joint changes in patients in whom the joint space increased after TNF- $\alpha$ blocker therapy. The JSW also increased after TNF- $\alpha$ blocker therapy in all 6 cases (The mean JSW values before and after TNF- $\alpha$ treatment were $0.88 \pm 0.79 \mathrm{~cm}$ and
$2.24 \pm 0.42 \mathrm{~cm}, \mathrm{p}=0.03$; Table 1). Patient 5 underwent computed tomography, which also showed increased hip joint space (Figure 1).

Patient characteristics are shown in Table 1 . The mean age at the time of anti-TNF- $\alpha$ blocker inception was $32 \pm 13.7$ years, and the mean disease duration was $11.2 \pm 6.1$ years. The mean duration of anti-TNF- $\alpha$ therapy was $1.97 \pm 1.3$ years. The anti-TNF- $\alpha$ inhibitors used varied among patients, and included infliximab (IFX), etanercept (ETN), and adalimumab (ADA). ADA and ETN were used in Patient 4 because of the low treatment efficacy of ETN.

The mean Bath Ankylosing Spondylitis Disease Activity Index (BASDAI) score decreased from 6.1 to 2.6 after anti-TNF- $\alpha$ inhibitor treatment and good clinical response was observed in all cases. All but Patient 3 had a high inflammatory burden with an elevated erythrocyte sedimentation rate (ESR) and C-reactive protein (CRP) level before anti-TNF- $\alpha$ therapy; the ESR and serum CRP level normalized following treatment.

An increase in hip joint space after anti-TNF- $\alpha$ treatment was observed in all 6 patients with AS in this case series.

There is 1 report of the restoration of hip joint space in patients with AS treated with IFX ${ }^{9}$. In addition, some reports showed no increase in hip joint space in AS, but did demonstrate inhibition of radiographic progression after use of an anti-TNF- $\alpha$ agent ${ }^{8,10}$. These findings suggest a positive effect with anti-TNF- $\alpha$ treatment on the osteodestructive changes that occur in spondyloarthritis, which are also known to occur in RA.

The first limitation of our study is that it is not known whether the increase in hip joint space was due to actual cartilage regeneration, because an increase in hip joint space in anteroposterior radiographs does not mean cartilage regeneration. More accurate imaging such as magnetic resonance imaging (MRI) is needed to evaluate cartilage itself. The second limitation is that the number of cases is too small to conclude that anti-TNF- $\alpha$ agents have a reparative effect on damaged cartilage of the hip.

Nevertheless, our study suggests that anti-TNF- $\alpha$ treatment reduces hip arthritis in AS. Prospective longterm studies using MRI are needed to obtain a reliable conclusion regarding the effect of TNF- $\alpha$ blockers on cartilage regeneration.

RAN SONG, MD, PhD, Assistant Professor, School of Medicine, Kyung Hee University, Division of Rheumatology, Department of Internal Medicine, Kyung Hee University Hospital at Gangdong; SANG WAN CHUNG, MD, Division of Rheumatology, Department of Internal Medicine, Kyung Hee University Hospital at Gangdong; SANG-HOON
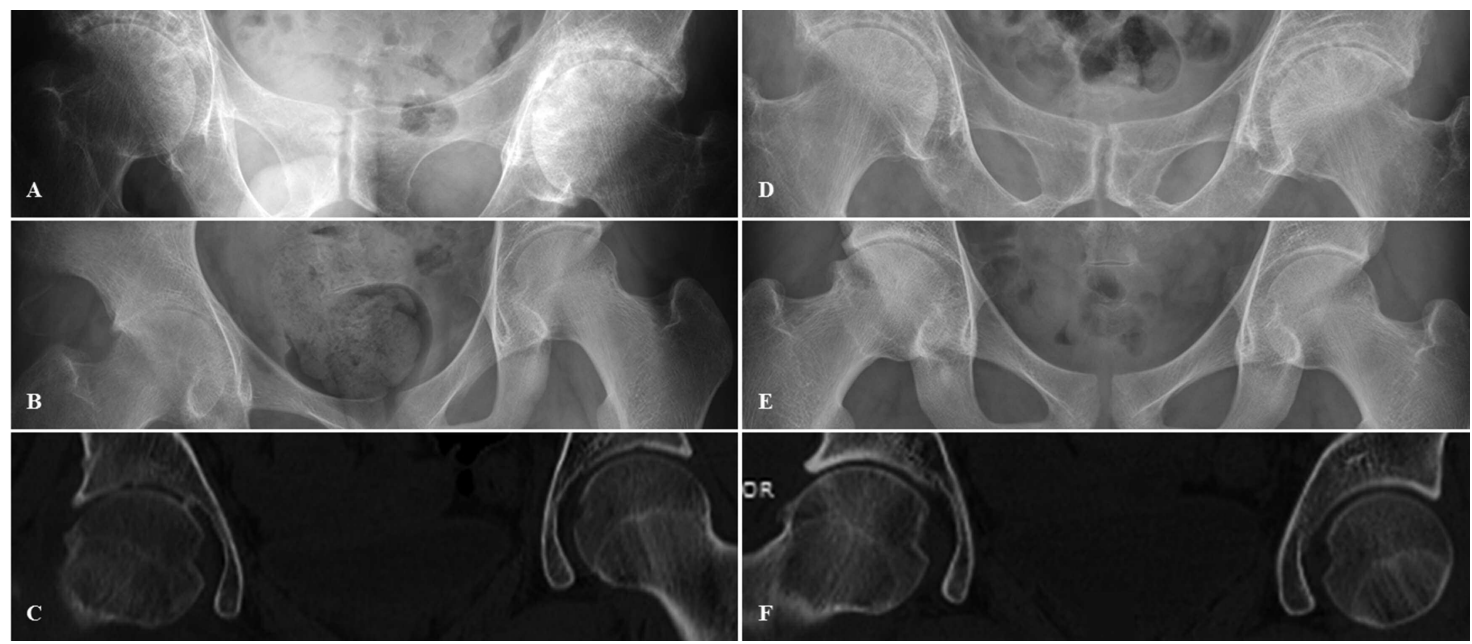

Figure 1. Hip joint changes in patients in whom the joint space increased after TNF- $\alpha$ blocker therapy. Images are hip radiographs of Patient 4 (A and D) and Patient 5 (B and E), and CT scans of Patient 5 (C and F). (A-C) Hip joint space before TNF- $\alpha$ blocker therapy. (D-F) Hip joint space after TNF- $\alpha$ blocker therapy. TNF: tumor necrosis factor; CT: computed tomography. 
Table 1. Characteristics of patients in whom hip joint space increased after anti-TNF- $\alpha$ treatment.

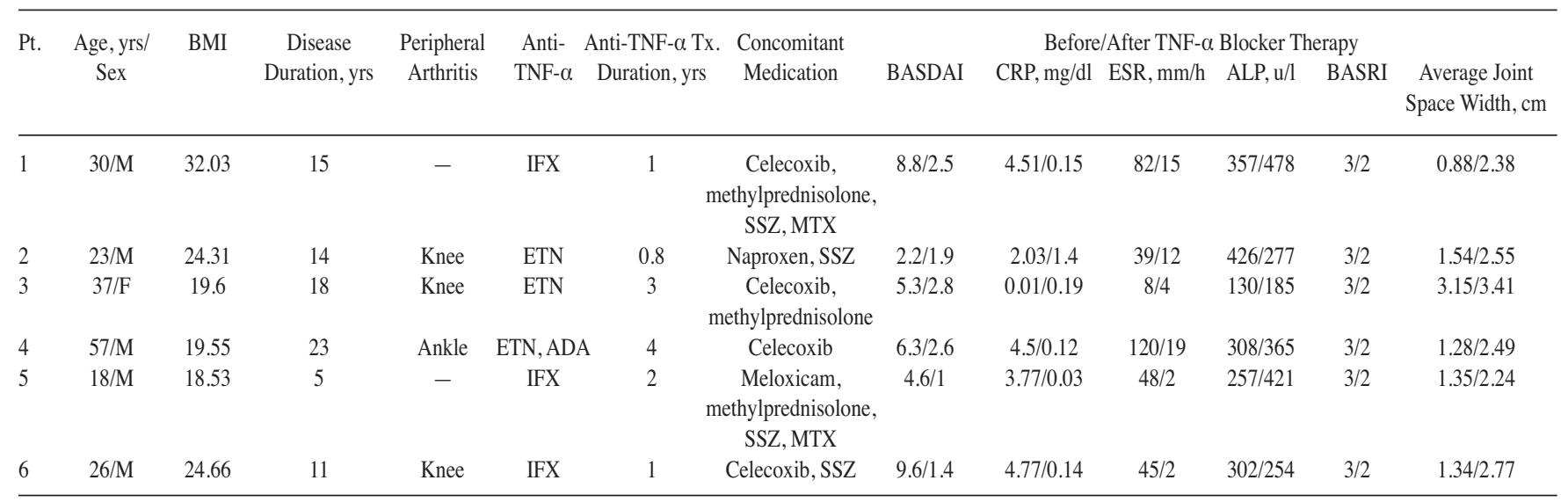

BMI: body mass index; TNF: tumor necrosis factor; Tx.: treatment; BASDAI: Bath Ankylosing Spondylitis Disease Activity Index; CRP: C-reactive protein; ESR: erythrocyte sedimentation rate; ALP: alkaline phosphatase; BASRI: Bath Ankylosing Spondylitis Radiology Index; SSZ: sulfasalazine; MTX: methotrexate; ETN: etanercept; IFX: infliximab; ADA: adalimumab.

LEE, MD, PhD, Associate Professor, School of Medicine, Kyung Hee University, Division of Rheumatology, Department of Internal Medicine, Kyung Hee University Hospital at Gangdong, Seoul, South Korea. Address correspondence to Professor S.H. Lee, Division of Rheumatology, Department of Internal Medicine, Kyung Hee University Hospital at Gangdong, School of Medicine, Kyung Hee University, 892 Dongnam-ro, Gangdong-gu, Seoul 134-727, Republic of Korea. E-mail:

boltaguni@yahoo.co.kr

\section{ACKNOWLEDGMENT}

The authors thank Dr. S.Y. Park for her help in interpretation of radiographs and computed tomography.

\section{REFERENCES}

1. Callhoff J, Sieper J, Weiss A, Zink A, Listing J. Efficacy of TNF alpha blockers in patients with ankylosing spondylitis and non-radiographic axial spondyloarthritis: a meta-analysis. Ann Rheum Dis 2015;74:1241-8.

2. Chen HA, Chen CH, Liao HT, Lin YJ, Chen PC, Chen WS, et al. Factors associated with radiographic spinal involvement and hip involvement in ankylosing spondylitis. Semin Arthritis Rheum 2011;40:552-8.

3. Russell AS, Rosenbaum JT. Anti-tumor necrosis factor therapies in immune-mediated rheumatic diseases. Other observations from the clinic. J Rheumatol Suppl. 2010 May;85:53-62.

4. Appel H, Kuhne M, Spiekermann S, Kohler D, Zacher J, Stein H, et al. Immunohistochemical analysis of hip arthritis in ankylosing spondylitis: evaluation of the bone-cartilage interface and subchondral bone marrow. Arthritis Rheum 2006;54:1805-13.

5. Chen WS, Chen CH, Lin KC, Tsai CY, Liao HT, Wang HB, et al. Immunohistological features of hip synovitis in ankylosing spondylitis with advanced hip involvement. Scand J Rheumatol 2009;38:154-5.

6. Revell PA, Mayston V. Histopathology of the synovial membrane of peripheral joints in ankylosing spondylitis. Ann Rheum Dis 1982;41:579-86.

7. MacKay K, Brophy S, Mack C, Doran M, Calin A. The development and validation of a radiographic grading system for the hip in ankylosing spondylitis: the Bath Ankylosing Spondylitis Radiology Hip Index. J Rheumatol 2000;27:2866-72.

8. Konsta M, Sfikakis PP, Bournia VK, Karras D, Iliopoulos A. Absence of radiographic progression of hip arthritis during infliximab treatment for ankylosing spondylitis. Clin Rheumatol 2013;32:1229-32.

9. Verbruggen G. Chondroprotective drugs in degenerative joint diseases. Rheumatology 2006;45:129-38.

10. Lian F, Yang X, Liang L, Xu H, Zhan Z, Qiu Q, et al. Treatment efficacy of etanercept and MTX combination therapy for ankylosing spondylitis hip joint lesion in Chinese population. Rheumatol Int 2012;32:1663-7.

J Rheumatol 2017;44:11; doi:10.3899/jrheum.161401 